\section{Cahiers de Narratologie}

Analyse et théorie narratives

29 | 2015

Street Art 1

\title{
Qu'est-ce que le Street art ? Essai et discussion des définitions
}

Ulrich Blanché

\section{(2) OpenEdition}

Journals

Electronic version

URL: http://journals.openedition.org/narratologie/7397

DOI: 10.4000/narratologie.7397

ISSN: 1765-307X

Publisher

LIRCES

\section{Electronic reference}

Ulrich Blanché, "Qu'est-ce que le Street art? Essai et discussion des définitions », Cahiers de Narratologie [Online], 29 | 2015, Online since 31 January 2016, connection on 10 December 2020. URL: http://journals.openedition.org/narratologie/7397 ; DOl : https://doi.org/10.4000/narratologie.7397

This text was automatically generated on 10 December 2020 .

Article L.111-1 du Code de la propriété intellectuelle. 


\title{
Qu'est-ce que le Street art? Essai et discussion des définitions
}

\author{
Ulrich Blanché
}

\section{Introduction}

1 Cedar Lewisohn, curateur de l'exposition de Street art à la Tate Modern (2008), déclarait dans une conférence internationale à Lisbonne en 2014, que les chercheurs spécialistes de Street art étaient plutôt des fans que des chercheurs critiques. Selon lui, la plupart des chercheurs s'occupant de Street art n'écrivent pas sans distance critique, mais la grande majorité des interventions lors de conférences scientifiques reste de l'ordre du descriptif. Ces contributions peuvent être résumées schématiquement par : «il existe un projet de Street art dans telle ville et je vais vous donner plus de détails sur cela»- et c'est tout. Nous décrivons souvent les projets de Street art, sans pourtant expliquer comment nous utilisons ce terme.

2 Quand Lewisohn affirme que la recherche sur le Street art est le fait de fans, il pourrait avoir raison pour ce qui est de la littérature anglo-saxonne. Cependant, il n'a certainement jamais lu de contributions sur le Street art en français, suédois, allemand ou italien. Par conséquent, la question des «fans » se révèlerait un simple problème de communication.

Qu'est-ce que c'est le Street art? Telle était la question des appels à contributions pour deux conférences, l'une à New York en mars dernier et l'autre à Nice, dont cet article est tiré. Depuis environ 2005, des chercheurs ont essayé d'y répondre. Plusieurs d'entre eux n'écrivent pas en anglais. Ma contribution est une courte introduction et discussion du terme "Street art", ainsi que des concepts liés à ce dernier, du point de vue d'un chercheur travaillant sur la culture visuelle. 


\section{Les graffitis}

4 Le terme graffiti, proche du Street art, est aujourd'hui associé à une forme particulière appelée style writing.

5 Cependant, le mot graffiti dérive du terme sgraffito, mentionné pour la première fois par Giorgio Vasari en $1564^{1}$. Le terme désignait la technique, liée à la peinture à fresco, du sgraffito ou griffage des façades des maisons durant la Renaissance. De ce fait, il ne s'agit pas d'un terme latin per se. Par ailleurs, aucun sgraffito n'a pas été utilisé pour des inscriptions officieuses sur les murs de Pompei. Ce phénomène n'apparaît que plus tard. Notre mot " graffiti », proche de l'italien «sgraffiare » ou « scrivere », commence à perdre son sens technique autour de 1850, en faveur de ses caractéristiques plus officieuses. L'étude de ces "graffitis » se révèle particulièrement pertinente pour des recherches en histoire ancienne et en archéologie, comme l'exemple de Raffaele Garrucci et son équipe d'archéologues découvrant Pompei peut en témoigner ${ }^{2}$. Les Romains n'avaient pas de terme pour désigner ce que les chercheurs du XIX siècle appelaient " graffiti ». De ce que nous savons, "l'écriture officieuse sur les murs» n'était pas interdite. Les Romains avaient une compréhension différente de ce qui était «public». Souvent, dans les conférences sur l'histoire du graffiti, sont montrés des clichés d'écritures sur les murs de Pompei, mais il ne s'agit en réalité pas de graffitis. Ce sont des peintures, habituellement rouges, des anciennes publicités ou slogans électoraux. Certains chercheurs confondent ces deux phénomènes quand ils débutent leur chronologie des graffitis.

Durant le XIX ${ }^{e}$ siècle, le mot "graffiti » se réfère aux mots écrits ou gravés. Le sens primaire des graffitis, c'est-à-dire dans le sens vasarien du terme, était celui d'images.

7 Les ouvrages de Johannes Stahl $(1989,1990,2009)$ fournissent une histoire des graffitis traditionnels (donc également une histoire du Street art) jusque dans les années 1980 ; tandis que, dans son ouvrage The G-Word (2014), Jacob Kimvall développe une discussion différenciée du style writing graffiti contemporain, non pas seulement en tant qu'art ou vandalisme. Stahl connaît la signification traditionnelle de graffitis. Il est toujours attaché à la définition élaborée par Garrucci, c'est-à-dire des signes officieux, soit style writing, graffitis des salles de bains du XVIII e siècle ou Street art contemporain.

\section{Le terme « Street art »}

8 Le pionnier du Street art John Fekner a donné une définition très large de celui-ci : « tout art dans la rue qui n'est pas des graffitis ${ }^{3}$ ", c'est-à-dire qui n'est pas du style writing. Je suis d'accord avec l'affirmation de Lewisohn disant que le Street art n'est pas synonyme de graffiti $^{4}$ - même si, comme nous le verrons, les genres se confondent parfois. Cependant, le Street art ne dérive pas dans son ensemble du graffiti writing, comme Lewisohn l'affirme ${ }^{5}$

D'après Claudia Walde, le Street art est devenu un mouvement collectif depuis 2000. Avant cette date, c'étaient des artistes seuls, tel que John Fekner, qui réalisaient des œuvres de ce que nous appelons aujourd'hui, rétrospectivement, le Street art. Gerard Zlotykamien, Harald Naegeli ou Jenny Holzer en sont d'autres exemples 6

10 Entre 2000 et 2005, ce que nous considérons aujourd'hui en tant que Street art n'était pas toujours dénommé ainsi. Julia Reineke (2007) explique que le terme fait une percée dans 
les médias en 2005. Autour de 2004, les termes post-graffiti ${ }^{7}$ et urban art rivalisaient entre eux (ainsi qu'avec quelques autres termes) pour la domination dans les forums de langue anglaise en ligne, où artistes et auteurs s'engageaient dans des discussions et controverses terminologiques ${ }^{8}$. Le terme "Street art » n'a donc pas été inventé seulement par des chercheurs mais par des artistes, chercheurs journalistes spécialisés en Street art et, plus généralement, en média.

11 Avant la domination de la dénomination «Street art ", chacun de ces termes conflictuels appuie sur des aspects différents de ce que l'on appelle «Street art » dix ans plus tard. «Post-graffiti » implique faussement que le graffiti soit quelque chose du passé ${ }^{\text {, alors }}$ que le Street art est en fait né du (style writing et d'autres formes de) graffitis, en particulier à la lumière des matériaux et médias utilisés, ainsi que des biographies des Street artists. Ni des artistes comme les French Pochoirists autour de Blek le Rat pendant les années 1980, ni le punk stenciling ne sont inclus dans les post-graffitis. Aujourd'hui, ce genre de stencil graffiti est vu à la fois comme précurseur et à la fois comme sous-catégorie du Street art, notamment du fait de la popularité des stencils tant dans les médias que parmi le public, même si les stencils ne représentent qu'une des techniques utilisées en Street art. À différence du style writing, le stencil graffiti est ancré dans le contexte historique des graffitis. Ce dernier est celui qui débute à Pompei et continue aujourd'hui, regroupant bathroom graffitis et citations dans les espaces publics. Chacune de ces pratiques s'inscrit dans la catégorie du daub scrabbling.

La signification de « Street art » a changé au fil du temps. En 1975, Robert Sommer utilisait le terme "Street art » pour indiquer les peintures murales, même si ce genre de projet est habituellement légal et constitue « une forme d'art et de communication visant tant les masses des habitants que les passants et qui est planifié et approuvé pour des expositions dans l'espace public ${ }^{10} »$. Outre le fait que ce genre d'art soit légal, ce public art n'est pas du Street art, puisqu'il est « une œuvre d'art contemporaine localisée en dehors des galeries et des musées, un objet communicatif qui démocratise l'accès à l'art moderne ${ }^{11}$ ». En 1996, la traduction anglaise d'un livre russe sur la propagande soviétique publié en 1984, fut intitulée Street Art of the Revolution: Festivals and Celebrations in Russia 1918-33. Même si une certaine forme de Street art aujourd'hui plonge ses racines dans la propagande et les posters politiques, soit de la Russie des années 1920, soit de l'Italie ou de l'Allemagne des années 1940 ou bien de la France des années 1960, la compréhension du Street art dans la période autour de 1990 diffère de l'usage du terme depuis 2005. Beaucoup d'objets de propagande n'étaient pas illégaux. En 1985, Allan Schwartzman publie un livre intitulé Street art. Sa compréhension du terme est proche de celle utilisée ici, même s'il publie des clichés mélangeant style writing graffiti légal et illégal avec ce qui aujourd'hui est appelé Street art.

\section{Définition du Street art}

13 Je suis d'accord avec Peter Bengsten (2014) sur le fait que «le terme "Street art" ne peut pas être défini de manière définitive, puisque ce qu'il désigne est constamment négocié ${ }^{12}$ ». Pour cela, la définition suivante du Street art n'est pas conclusive :

Le Street art consiste en des images auto-autorisées, personnages et formes créés ou appliqués aux surfaces de l'espace urbain, qui cherchent à communiquer avec un vaste public. Le Street art est réalisé d'une façon performative et in situ (site-specific), 
éphémère et participative. Dans la plupart des cas, elle est vue sur Internet. Elle diffère des graffitis et du Public art.

\section{Street (et) art}

14 La faiblesse de ma définition, comme la faiblesse du terme "Street art ", repose dans la portion concernée par la partie « art ». Selon Ilaria Hoppe (2009), le Street art ne s'est pas souvent détaché du champ de l'art, comme c'était en revanche le cas en ce qui concerne la forme ${ }^{13}$. Pour cela, ni Street art ni graffitis n'ont à être catégorisés comme art, même si certains chercheurs comme Nora Schmidt ${ }^{14}$ (2009) ou l'artiste John Fekner le font. Quand je parle d'" images, caractères et formes ", je dois, en conséquence, comme Norbert Siegl, catégoriser les annonces pour animaux perdus ${ }^{15}$ en tant que Street art ou simplement parler d'«installation auto-autorisée de dessins artistiques et signes quelconques». Je suis hostile à tout cela, puisque le Street art reflète souvent les sensibilités optiques et/ou techniques du dessin graphique ou des illustrations plutôt que des soi-disant beaux-arts. Je ne vois pas le Street art en tant qu'historien de l'art mais en tant que scientifique des images («Bildwissenschaftler »), qui travaille avec des images de toute sorte, sans prêter attention à leur rapport avec l'étiquette problématique d' « art ».

Johannes Stahl échappe à ce dilemme de «Street art égal art» en se référant au photographe, auteur et artiste Brassaï. Ce dernier nommait en 1933 le scratch-graffiti, plus proche des graffitis à Pompéi que de ceux dans les rues de Paris à son époque, d' " art bâtard des rues malfamées ${ }^{16}$ ». Cette conception avant-la-lettre de Street art proposée par Brassaï fonctionne d'une façon antithétique. Pour lui, rue et art sont des valeurs également opposées, un «mutt ${ }^{17}$ » ou « mongrel », comme le définit Johannes Stahl dans son manuel. «Rue » (dans le sens de «street») n'est pas une qualification de «art ». Bien au contraire, elle en est plutôt l'opposé, comme dans le cas du terme "anti-art». Le dialogue entre l'antonyme rue et art est globalement constructif, même si dans certains cas d'une façon destructive. "Street art » peut se référer à des phénomènes du quotidien se déroulant dans la rue et qui sont perçus en tant qu' " art ", qu'ils soient conçus comme tel ou pas. L'art est dans les yeux de l'observateur, comme c'était le cas avec Brassaï, qui découvrait l'art dans la rue, utilisait ses yeux (ou une caméra) pour couper ces œuvres de leur contexte et en faisait des objets d'art ${ }^{18}$, qui pourtant préservent la sensation et l'authenticité de leur site. En bref, certaines formes de Street art sont plus Street, d'autres plus art. Dans cet esprit, Jacob Kimvall (2014) parle de « vandalisme de graffiti et graffiti $\operatorname{art}^{19} »$.

\section{« Illégal » versus « auto-autorisé »}

Le terme « espace urbain » dans la définition précédemment citée est analogue à celui employé par Norbert Siegl, selon qui « les surfaces appartiennent à d'autres [...] ou à la juridiction publique ${ }^{20}$ ». Cela exclue intrinsèquement des espaces concédés à des artistes de rue ou des espaces où ces artistes sont autorisés à créer leurs œuvres. L'espace, la rue du Street art dicte une illégalité nécessaire, du moins en Europe et aux États-Unis.

17 Tant les graffitis que le Street art sont liés par le fait d'être officieux ${ }^{21}$, non sollicités ${ }^{22}$, de n'avoir été ni commandés ni validés ${ }^{23}$, et donc, par conséquent, d'un point de vue légal, d'être souvent considérés comme actes de vandalisme. Comme les lois diffèrent de pays en pays, le Street art peut ne pas être illégal, par exemple en Chine ou en Amérique du 
Sud, tandis qu'il l'est en Europe ou aux États-Unis. Comme « illégal » ou «illicite » (avec une composante morale à côté de la composante légale) ne sont pas des termes pertinents pour le Street art, « auto-autorisé » pourrait donc être une définition plus appropriée.

La « nature auto-autorisée » de l'acte de réaliser une œuvre d'art est un commentaire sur le capitalisme et le consumérisme en général, dans la mesure où, initialement, elle ne peut pas fonctionner en termes de ventes marketing et est de ce fait autonome - dans le sens d'opposé à « l'art de galerie », où les artistes espèrent vendre ${ }^{24}$.

L'art officiel public est commandé ou installé avec le consentement du propriétaire ou déclaré légal rétroactivement par le propriétaire $d u$ site où il se trouve. La compréhension plus ou moins étroite du terme "Street art " dépend de sa future utilisation commerciale. Ceux qui ont créé de l'art en fonction d'une commande se trouvent rapidement critiqués d'avoir été influencés par un commanditaire en réalisant les désirs de ce dernier, au lieu de concevoir librement et de manière créative leur propre art.

«Street art » au sens propre s'applique à tout art dans l'espace urbain n'étant pas limité par la loi ou par le goût d'autorités tels que sponsors, propriétaires d'immeubles ou même par l'État - art qui n'est donc pas directement commercial. Ceci renforce le fait que l'artiste renonce à utiliser son travail à des fins commerciales, afin d'éviter que les puristes de l'art l'accusent de s'engager dans l'autopromotion (même si c'est presque toujours le cas). De cette manière, les Street artists se débranchent (théoriquement) du circuit des consommateurs et peuvent interagir avec un sujet artistique sans se corrompre avec les doubles standards : ils critiquent la culture de consommation, mais, en même temps, du moins indirectement, ils promeuvent leurs propres œuvres d'art, c'est-à-dire ils produisent de l'art vendable ${ }^{25}$. La plupart des artistes se voient confrontés tôt ou tard avec un conflit, le soi-disant «sell-out » conflict. D'un côté les artistes veulent vivre de leur propre art, de l'autre ils peuvent perdre leur propre crédibilité de la rue, c'est-à-dire leur réputation parmi leurs homologues ${ }^{26}$.

21 Norbert Siegl ${ }^{27}$, de la même manière que le ton général des médias, inclut dans son concept de "Street art» les phénomènes légaux tels que des stickers préalablement approuvés ou les images légales (commandées) sur les murs, œuvres d'artistes qui autrement travaillent illégalement. Cependant, je soutiens le refus d'Heike Derwanz (2013) de la définition très large de «Street art» qui inclut des aspects contredisant les valeurs primordiales du Street art en tant qu'art officieux et auto-autorisé, mais pas à cause de l'aspect commercial du Street art légal.

Dans un contexte de Street art le terme «mural » se réfère souvent à « des peintures de grand format, multichromatiques ainsi que des peintures de laboratoire tels que celles peintes avec pistolet et bombe de peinture ${ }^{28} »$. L'art mural est le plus souvent validé. Jean Baudrillard reconnaissait déjà qu'il existe d'énormes différences entre style writing et peintures murales. Ces dernières sont en effet liées à leurs propres communautés, ce sont des exemples d'art public, tandis que les graffitis et le Street art constituent plutôt des messages individuels qui peuvent être le résultat d'un petit groupe, mais ne bénéficient pas d'un accord officiel (même si cela peut se produire au cours du temps ${ }^{29}$ ). 


\section{Mot versus image} ornementale le fait dans le style writing. Dans le cas de ce dernier, les œuvres cherchent à communiquer entre elles plutôt qu'avec un public ${ }^{33}$, tout en créant un contraste direct avec la définition de Street art évoquée plus haut (p. 4: «[un art qui cherche] intentionnellement la communication avec un cercle étendu d'individus »). Même le Street art basé sur un usage plus important des mots est plus prompt à la communication que le Style Writing dans la priorité donnée à la lisibilité par le public. Baudrillard (1975) voit la signification politique des graffitis (même si, dans ce cas, il se réfère au style-writing ) dans la perturbation que ces derniers causent dans le système de signes et de communication écrite dans la ville, notamment à cause de l'absence de signification du contexte $^{34}$. La seule existence du style writing graffiti provoque des questions concernant tant la propriété que le fait de savoir qui a le droit de communiquer quelque chose et d'où le faire ${ }^{35}$. Cela constitue un point commun entre Street art et graffitis.

Même dans le cas où une peinture murale n'est ni autorisée ni commandée, il est très peu probable qu'elle soit considérée comme du vandalisme, parce qu'elle est plus aisément comprise et accessible au grand public que le graffiti writing. Cette grande compréhension s'explique par le fait que peintures murales et Street art sont des images et non pas des mots (illisibles). aux graffiti historiques qu'au style writing graffiti : «certainement, les graffitis peuvent être distingués de la littérature canonique dans le sens où il s'agit de matériel empathique, fixé dans le temps et l'espace, et d'objets plutôt que de mots ${ }^{30}$ ». L'aspect matériel et la solidité tendent à une compréhension d'un objet pictural et moins d'un texte. Même les Bathroom Graffitis cherchent à transmettre un message, par conséquent ils sont techniquement impossibles à distinguer du Street art. Cependant, les Bathroom Graffitis peuvent être plus proches de la "Street» que de l'«art». Stencils et images murales sont souvent moins cryptiques que le style writing. De surcroît, le pochoir a un élément communicatif de clarté et reproductibilité que permet sa lisibilité.

tendent plutôt vers les lettres écrites, tandis que le Street art tend vers une communication picturale. Cependant, les deux existent et évoluent dans le même continuum d'écriture et d'images ${ }^{31}$. Hoppe (2009) précisait que les œuvres de Street art sur papier sont même plus picturales que celles vaporisées sur une surface ${ }^{32}$.

Pour Lewisohn (2008), le Street art est moins limité que le style writing graffiti en ce qui concerne les règles stylistiques et les matériaux artistiques, comme les bombes de peinture et les marqueurs. La prise de position de Lewisohn peut être confirmée, du moins de manière générale, même si des exemples contredisant son affirmation sont aisément repérables, tant $\mathrm{du}$ point de vue qualitatif que quantitatif ${ }^{36}$. Les mêmes avertissements s'appliquent à une autre thèse de Lewisohn, qui considère le Street art comme une pratique plutôt de «studio » et non pas en tant que création techniquement ambitieuse et travail manuel dangereux dans la rue.

En général, nous pouvons affirmer que le Street art valorise et insiste souvent plus sur le contenu et la communication, tandis que le style writing exalte la virtuosité technique. 


\section{Le Street art est in-situ}

29 œuvre est à considérer en tant que Street art si, et seulement si, son usage matériel de la rue est intrinsèque à sa signification ${ }^{37}$ ». Comme Bengsten le remarque (2014), le problème de la définition de Riggle est le suivant : « Il n'est pas clair de savoir qui est tenu à juger si l'usage de la rue est en effet essentiel pour la signification d'une œuvre d'art spécifique ». Le contexte de chaque œuvre de Street art, c'est-à-dire "son usage matériel de la rue ", change pendant son existence comme dans un palimpseste. Si quelqu'un attache quelque chose à côté d'une œuvre qui s'y réfère, un objet sans connexion préalable avec le mur peut en trouver une après un certain moment.

La site-specificity du Street art est partie intégrante de la définition de Riggle. Par ailleurs, elle avait déjà été soulignée par Lewisohn (2008), Wacklawek (2008) et Hoppe (2009). Cependant, dans le Street art tout n'est pas in situ de la même manière. Si certaines œuvres sont faites sur mesure pour leur emplacement, d'autres pourraient être n'importe où dans la rue - comme une affiche. Il existe différents degrés de site-specificity d'une œuvre d'art. Tant les degrés que la qualité de site-specificity de l'œuvre peuvent changer au cours de la "vie d'étagère » de l'œuvre de rue. Cette dernière peut se référer à un certain mur, mais aussi à une certaine zone, rue, ville, pays ou toutes ces catégories ensemble.

\section{L'aspect performatif du Street art}

Derwanz attire l'attention à l'aspect performatif de la peinture murale (légale) ou de l'art sur le sol, qu'elle n'attribue pas au Street art ${ }^{38}$. Même s'il est très rare d'assister à la performance, à la mise en place souvent spectaculaire du Street art, je vois, comme l'indique Waclawek (2008), un élément performatif intégré dans chaque œuvre de Street art. « Le message, court et pointu, souvent marqué par des combinaisons inattendues [du Street art], transmet une qualité visuelle créée à toute vitesse ${ }^{39} \%$. Ce message visuel dicte la création des œuvres. Dans les mots de Banksy : « Le graffiti est un art où le geste est au moins aussi important que le résultat, voire plus. J'ai lu qu'un critique a décrit Jackson Pollock comme un artiste de performance qui se trouvait à utiliser de la peinture. La même chose pourrait être dit des auteurs de graffitis - artistes de performance qui se trouvent à utiliser de la peinture. Et enfreignent la loi ${ }^{40} »$. Tant dans le Street art que dans d'autres formes d'art public, il ne s'agit pas de performance, du moins d'après l'usage usuel du terme. Généralement, un performer exécute devant un public. Mais les œuvres de Street art transmettent souvent le message que « quelque chose s'est passé » sur le lieu où elles se trouvent. Ce « quelque chose » est très souvent l'attachement illégal d'une œuvre de Street art sur une surface dans un espace public. Son attachement aventureux, impertinent et hardi est partie intégrante de l'œuvre d'art. Certains Street artists ont adopté l'importance du temps des performances «classiques»-c'est-à-dire que le spectateur voit dans quel court délai l'artiste exécute l'œuvre sans être attrapé - ainsi que le caractère éphémère et l'importance de l'espace. Comme beaucoup de performances, leur art est adapté à un endroit particulier et perd sa condition originale lors d'un transfert. Comme beaucoup de performers, le Street artist vend des documentations, estampes, DVDs et livres de son Street art. Comme beaucoup de performers, il le fait dans un esprit ironique, en tant que souvenirs ou relique. Le Street art, comme performance, est un art basé sur un processus. Chaque œuvre d'art est imprimé avec des traces de ce 
processus. Tant dans le cas du Street art que dans celui de la performance, il s'agit de formes d'art qui ne sont pas rentables en elles-mêmes. Elles représentent des formes de consommation critique d'art, vu leur critique de l'objet d'art en tant qu'objet à consommer. Et l'aspect de la performance et celui de la site-specificity ont leur origine dans la situation spatiale, du lieu de naissance de l'œuvre, c'est-à-dire la rue. Les différentes formes de graffitis contiennent également cet élément performatif. Ce dernier a son origine dans la relation avec le lieu où l'œuvre est créée et dans la valeur, c'est-àdire dans le degré de danger physique ou légal que possède le lieu où est placée l'œuvre. Cet élément diffère du contenu de l'emplacement postulé par Lewisohn, selon lequel «le graffiti est un genre tellement obstiné qu'il refuse de s'attaquer aux conditions de sa localisation $^{41} »$.

\section{Le Street art est éphémère et participatif}

Le Street art est souvent participatif, c'est-à-dire que n'importe qui peut le repeindre, détruire, ajouter quelque chose ou le compléter. D'autres Street artists, des propriétaires et les mairies sont les acteurs usuels de changement ou d'effacement du Street art. Cependant, même le public peut devenir partie active d'une œuvre de Street art. Celle-ci n'est pas commandée et, par conséquent, non seulement hors-la-loi, mais peut aussi être altérée. Les panneaux d'affichage et l'art public ${ }^{42}$ sont réalisés sur commande et donc destinés à rester inaltérés. Le Writing graffiti a des règles très strictes sur qui est autorisé à altérer des œuvres d'autres style writers. Certains Street artists encouragent l'interaction avec d'autres dans la rue, tandis que d'autres essaient d'empêcher l'altération de leurs œuvres, par exemple grâce à l'usage de matériaux solides, tels les carreaux de l'artiste français Space Invader ou les sculptures métalliques de l'artiste new-yorkais Darius Jones. Dans ces cas, il s'agit d'une contre-réaction, puisque la participation à ces œuvres est destructive.

Mais il existe un autre type de participation non destructive en Street art, liée au rôle de la photographie. Plusieurs œuvres récentes ont un espace vide au milieu d'elles-mêmes, grâce auquel le public a la possibilité de poser pour une photographie ou un selfie.

La plupart des œuvres de Street art sont éphémères, c'est-à-dire temporelles. Le vent, le temps, le soleil et la pluie détruisent la plupart des œuvres non protégées. La «vie d'étagère » d'une œuvre de Street art varie, mais après quelques heures, mois ou années, elle cesse d'exister. La photographie peut aider à documenter le processus de changement d'une œuvre au fil du temps, processus souvent encouragé par plusieurs artistes.

Les Street artists utilisent également un aspect différent impliquant la participation: le rôle de la photographie (numérique) et celui du spectateur tant dans la rue qu'en ligne. Avec leur smartphones, les spectateurs en ligne de Street art rejoignent ceux dans la rue. Par exemple, cela s'est vérifié lors que Banksy géolocalisait les photos de ses œuvres sur Instagram, afin que ses fans puissent y aller et prendre une photo ou simplement voir l'œuvre. Les Street artists sont intéressés à faire participer les spectateurs dans leur art à travers une sorte de jeu de piste. Telle est la conséquence de l'inclusion, sur le site de Banksy au début de sa carrière, dans ses livres ou dans les magazines publiant ses entretiens, d'instructions pour attacher des pochoirs dans la rue. Les Street artists ne veulent pas seulement que l'on assiste à leur performance, ils invitent leur public à les rejoindre dans le jeu de rue. 


\section{Art urbain} inclue aussi des œuvres légales. «Art urbain » semblait un terme plus approprié pour le titre de cette étude, puisqu'il regroupe les différentes formes d'art : Street art, style writing ou art mural. Art urbain, dans un sens plus étroit que celui employé dans cet article, était et continue à être un synonyme de Street art. Les enchères Bonhams appelaient «Urban Art » leur vente d'œuvres de Street artists ou d'artistes qui souvent travaillent dans la rue. L'art urbain est une forme souvent pratiquée par des Street artists pour gagner leur vie. Fréquemment, ils remploient motifs ou des techniques tirés de leurs œuvres de Street art, sans l'illégalité ou l'auto-autorisation et souvent sans l'aspect de site-specificity.

Si des artistes de rue produisent des œuvres illégales dans la rue, ils deviennent des Street artists ou des graffiti writers (à nouveau). À la différence de la majorité des formes d'art public ou d'art dans l'espace public, l'art urbain se réfère stylistiquement au Street art ou au graffiti style writing, qui comptent sur la «crédibilité de rue » du Street art sans être illégal ou non-commissionné et sans s'appuyer sur l'apparence souvent peu audacieuse de l'art mural communautaire «sanctifié ». À la différence de l'art public, l'art urbain peut être exposé dans un musée ou dans une galerie et peut donc être vendu. Déplacées de la rue, des œuvres de Street art peuvent devenir des exemples d'art urbain. À différence du Street art et du Land art, la majorité de l'art urbain se concentre moins sur l'environnement urbain. L'art urbain est très souvent de l'art de galerie dans le style du Street art.

\section{Perspective}

En tant que chercheur en culture visuelle, engagé dans la recherche académique sur le Street art, je déplore l'absence d'un débat et d'une analyse de ce nous voyons. Plusieurs chercheurs s'occupent simplement des contours du Street art, c'est-à-dire les artistes, les scènes, la gentrification et la commercialisation. D'autres montrent plusieurs exemples et images de Street art, tout en laissant les spectateurs seuls avec cela, comme si les images pouvaient parler d'elles-mêmes. En effet, elles le font, mais chacune d'une façon différente. Plusieurs chercheurs ont interviewé des Street artists et graffiti writers, mais ils n'ont pas interagi avec le Street art lui-même, d'une façon visuelle. Par ailleurs, les entretiens ne sont pas la solution pour tout. « Mais c'est l'artiste qui l'a dit ! - bien sûr, mais, il pourrait avoir menti juste pour mieux se mettre en valeur. Nous devons traiter les entretiens non pas en tant que vérité absolue, mais en tant que source. Je plaide pour plus de recherche sur le Street art et moins de recherche sur les artistes eux-mêmes, tout en étant conscients que l'un ne fonctionne pas sans l'autre. 


\section{NOTES}

1. "E questo è il lavoro che per esser dal ferro graffiato, hanno chiamato i pittori sgraffito. ", d'après Vasari, G. (1966-1987). " Degli Sgraffiti delle case che reggono a l'acqua, quello che si adoperi a fargli, e come si Iavorino le grottesche nelle mura. ». In: Barocchi, P. ; Bettarini, P. (éd.) Le vite dei più eccellenti Pittori, Scultori e Architetti. Florence : S.P.E.S., Libro I, Capitolo XXVI.

2. D'après Stahl, J. (1990) Graffiti: Zwischen Alltag und Ästhetik. Munich : Scaneg, p. 5. Il se réfère à la définition de graffitis de W.P. McLeans dans la Encyclopaedia Universalis. Paris: Encyclopaedia Universalis (1968), vol. 7, p. 850.

3. John Fekner cité dans Lewisohn, C. (2008) Street art. The Graffiti Revolution. catalogue de l'exposition à la Tate Modern. Londres: Abrams, p. 23.

4. Lewisohn, C. (2008) Street art. The Graffiti Revolution. catalogue de l'exposition à la Tate Modern. Londres : Abrams, p. 15-16.

5. Lewisohn, C. (2008) Street art. The Graffiti Revolution. catalogue de l'exposition à la Tate Modern. Londres : Abrams, p. 15-16.

6. Walde, C. (2008) Sticker City. Paper Graffiti Art. Londres: Thames and Hudson.

7. Reinecke, J. (2007) Street-Art. Eine Subkultur zwischen Kunst und Kommerz. Bielefeld: transcript; Waclawek, A. (2008) From Graffiti to the Street art Movement: Negotiating Art Worlds, Urban Spaces, and Visual Culture, c. 1970-2008. [Thèse de doctorat à l'Université Concordia de Montreal, Québec] http://spectrum.library.concordia.ca/976281/1/NR63383.pdf (consulté le 12.03.2015). Les deux auteures évidemment préféraient le terme Post-Graffiti mais ont modifié leurs titres et les termes en Street art, puisque ce dernier est devenu plus courant dans les médias.

8. Reinecke, J. (2007) Street-Art. Eine Subkultur zwischen Kunst und Kommerz. Bielefeld: transcript, p. 13-17.

9. D-Face cité dans Reinecke, J. (2007) Street-Art. Eine Subkultur zwischen Kunst und Kommerz. Bielefeld : transcript, p. 16.

10. Derwanz, H. (2013) Street art-Karrieren. Neue Wege in den Kunst- und Designmarkt. Bielefeld: transcript, p. 112.

11. Danko, D. (2009) Wenn die Kunst vor der Tür steht. Ansätze zu notwendigen Differenzierungen des Begriffs „Kunst im öffentlichen Raum“. kunsttexte.de 01/2009. http://edoc.hu-berlin.de/ kunsttexte/2009-1/danko-dagmar-0/PDF/danko.pdf (consulté le 15.1.2015), p. 2.

12. Bengtsen, P. (2014) Street art World. Lund : Almendros de Granada Press, p. 11.

13. Hoppe, I. (2009) Street art und ,Die Kunst im öffentlichen Raum'. kunsttexte.de 01/2009. http:// edoc.hu-berlin.de/kunsttexte/2009-1/hoppe-ilaria-6/PDF/hoppe.pdf (consulté le 15.1.2015), p. 5.

14. Nora Schmidt (2009) in Street art. Legenden zur Straße. Berlin : Archiv der Jugendkulturen Verlag KG, p. 86.

15. Siegl, N.; Schaefer-Wiery, S. (éd., 2009) Der Graffiti Reader. Essays internationaler Experten zum Kulturphänomen Graffiti. Vienne : graffiti edition, p. 68. Cependant, les annonces d'animaux perdus sont utilisées comme base pour des œuvres de Street art de temps en temps.

16. Brassaï, « Du mur des cavernes au mur d'usine ». In: Minotaure, 3/4, 1933, p. 6-7.

17. Stahl, J. (2009) Street art. Potsdam : Ullmann publishing, p. 7.

18. Wucherer, B. (1989) «Auch kannst du allerlei Schlachten sehen - Künstlerische Inspirationen vor der Wand » In: Stahl, J. (éd.) An der Wand. Cologne : Dumont, p. 145.

19. Kimvall, J. (2014) The G-word: Virtuosity and Violation, Negotiating and Transforming Graffiti. Stockholm : Dokument Press, p. 11. 
20. Siegl, N. ; Schaefer-Wiery, S. (éd., 2009) Der Graffiti Reader. Essays internationaler Experten zum Kulturphänomen Graffiti. Vienne : graffiti edition, p. 67.

21. Krause, D. ; Heinicke, C. (2006) Street art. Die Stadt als Spielplatz. Berlin Archiv der Jugendkulturen Verlag KG, p. 58.

22. Siegl, N. ; Schaefer-Wiery, S. (éd., 2009): Der Graffiti Reader. Essays internationaler Experten zum Kulturphänomen Graffiti. Vienne : graffiti edition, p. 67.

23. Bengtsen, P. (2014) Street art World. Lund : Almendros de Granada Press, p. 131.

24. Du moins pas dans le court terme. Non seulement dans le cas de Banksy, mais aussi dans celui de Fairey, leurs œuvres dans la rue ont influencé la hausse de vente de leurs estampes et livres.

25. Voir la note de bas de page précédente. Dans le cas du Street art de Bristol et Londres, celui-ci, jusqu'à un certain point, renforce le tourisme.

26. Les premières allégations contre Banksy apparaissent dès 2003. Voir Beale, S. (2004) « First Against the Wall ». In: Arena Magazine, Janvier 2004, p. 152-157.

27. Siegl, N. (2005/06) "Street art ». In: Brockhaus Enzyklopädie, 21. Edition, Mannheim: Brockhaus.

28. Axel Philipps, Eelco Herder et Sergej Zerr incluent des graffitis et des œuvres d'art légales réalisés par des Street artists dans une communication non publiée ("The recognition and dissemination of Street art with Flickr. A visual content analysis", 2013) cité par Bengtsen, P. (2014) Street art World. Lund : Almendros de Granada Press, p. 131, 210.

29. Telle que l'œuvre de Banksy sélectionnée à Bristol en 2007, "Mild Mild West ", qui est devenue un point de repère dans le monde de l'art alternatif. Voir http://www.bbc.co.uk/bristol/ content/articles/2007/05/29/alternativelandmark_winners_feature.shtml (20 Nov 2014)

30. Milnor, K. (2014) Graffiti and the Literary Landscape in Roman Pompeii. Oxford - New York : Oxford University Press, p. 3.

31. Klitzke, K. Street art als kulturelle Praxis. [Mémoire de recherché non publié, Université Humboldt de Berlin, Berlin 2005], p. 24. Voir aussi Krause, D.; Heinicke, C. (éd., 2006) Street art. Die Stadt als Spielplatz. Berlin Archiv der Jugendkulturen Verlag KG, p. 60 ou Lewisohn, C. (2008) Street art. The Graffiti Revolution. catalogue de l'exposition à la Tate Modern. Londres : Abrams, p. 23.

32. Ilaria Hoppe dans Street art. Legenden zur Straße. Berlin : Archiv der Jugendkulturen Verlag KG, p. 100.

33. Street art collective Faile cité dans Lewisohn, C. (2008) Street art. The Graffiti Revolution. catalogue de l'exposition à la Tate Modern. Londres : Abrams, p. 15.

34. Baudrillard, J. (1978): Kool Killer oder der Aufstand der Zeichen. [original français de 1975] Berlin : Merve Verlag, p. 30.

35. Krause, D. ; Heinicke, C. (2006) Street art. Die Stadt als Spielplatz. Berlin Archiv der Jugendkulturen Verlag KG, p. 9.

36. Par exemple: Le light painting (peinture de lumière), les digital graffiti (graffiti numérique), les graffitis 3D, ou les fire extinguisher graffiti (graffiti à l'extincteur).

37. Riggle, N. (2010) « Street Art: The Transfiguration of the Commonplaces ». In The Journal of Aesthetics and Art Criticism, 68.2, pp. 243-257.

38. Derwanz, H. (2013) Street art-Karrieren. Neue Wege in den Kunst- und Designmarkt. Bielefeld : transcript, p. 110, 120.

39. Beck, H. (éd., 2004) Graffiti. Stuttgart : Reclam, p. 5.

40. Banksy a été interviewé par Hamilton Keegan, cf. Keegan, H. (2013) «Village Voice Exclusive: An Interview With Banksy, Street art Cult Hero, International Man of Mystery ». In Village Voice, Oct. 9, 2013. http://www.villagevoice.com/2013-10-09/art/banksy-better-out-than-in-new-yorkresidency-Street-art-Graffiti/full/ (02.03.2015).

41. Lewisohn, C. (2008) Street art. The Graffiti Revolution. catalogue de l'exposition à la Tate Modern. Londres : Abrams, p. 63. 
42. Pour la différence entre l'art urbain et l'art public voir Hoppe, I. (2009) Street art und ,Die Kunst im öffentlichen Raum'. kunsttexte.de 01/2009. http://edoc.hu-berlin.de/kunsttexte/2009-1/hoppeilaria-6/PDF/hoppe.pdf (consulté le 15.1.2015), et Danko, D. (2009) Wenn die Kunst vor der Tür steht. Ansätze zu notwendigen Differenzierungen des Begriffs „Kunst im öffentlichen Raum“. kunsttexte.de 01/2009. http://edoc.hu-berlin.de/kunsttexte/2009-1/danko-dagmar-0/PDF/danko.pdf (consulté le 15.1.2015). Voir aussi Bengtsen, P. (2014) Street art World. Lund : Almendros de Granada Press, chapitre 4.

\section{ABSTRACTS}

Cedar Lewisohn, curator of the Street Art show at Tate Modern (2008), blustered in an international street art conference in Lisbon (2014) about Street Art academics being rather fans than critical academics. Most Street Art researchers may not write without critical distance, but most papers given at Street Art conferences are rather descriptive. We often just describe Street Art projects, often without explaining how we use the term Street Art.

When Lewisson says, Street Art research is just written by fans he might be right for what he reads. I impute to Lewisohn that he hardly ever read any Street Art academic writing that is not English but French, Swedish, German or Italian. So this whole "fan thing " might be just a problem of communication.

«What is Street Art?». This was the first question in a call for Paper for a Street Art conference in New York in March as well as in the Nice conference. Since about 2005, a range of academic authors already tried to answer that question. A lot of them do not write in English. My paper is a short introduction and discussion of the term Street Art and related terms from the position of a visual culture researcher. First and foremost I discuss the following attempt of a definition:

Street Art consists of self-authorized pictures, characters, and forms created in or applied to surfaces in the urban space that intentionally seek communication with a larger circle of people. Street Art is done in a performative and often site-specific, ephemeral, and participatory way. Street Art is mostly viewed online. It differs from Graffiti and Public Art.

Cedar Lewisohn, curateur de l'exposition de Street art à la Tate Modern (2008), déclarait dans une conférence internationale à Lisbonne en 2014, que les chercheurs spécialistes de Street art étaient plutôt des fans que des chercheurs critiques. Selon lui, la plupart des chercheurs s'occupant de Street art n'écrivent pas sans distance critique, mais la grande majorité des interventions lors de conférences scientifiques reste de l'ordre du descriptif. Les projets de Street art sont souvent décrits sans qu'il n'y ait une tentative d'explication de ce terme.

Quand Lewisohn affirme que la recherche sur le Street art est le fait de fans, il pourrait avoir raison pour ce qui est de la littérature anglo-saxonne. Cependant, Lewisohn n'a probablement jamais lu de contributions sur le Street art en français, suédois, allemand ou italien. Par conséquent, la question des «fans " se révèlerait un simple problème de communication.Qu'estce que c'est le Street art ? Telle était la question des appels à contributions pour deux conférences, l'une à New York en mars dernier et l'autre à Nice, dont cet article est tiré. Depuis environ 2005, des chercheurs ont essayé d'y répondre. Plusieurs d'entre eux n'écrivent pas en anglais. Ma contribution est une courte introduction et discussion du terme Street art, ainsi que des concepts liés à ce dernier, du point de vue d'un chercheur travaillant sur la culture visuelle. Je discuterai la tentative suivante de définition: le street art consiste en des images auto-autorisées, 
personnages et formes créés ou appliqués aux surfaces de l'espace urbain, qui cherchent à communiquer avec un vaste public. Le Street art est réalisé d'une façon performative et in situ ( site-specific), éphémère et participative. Dans la plupart des cas, il est vu sur Internet. Il diffère des graffitis et du Public art.

\section{INDEX}

Chronological index: XXIème siècle

Geographical index: monde

Mots-clés: Banksy, art urbain, définition, recherche

Keywords: Street Art, Graffiti, Urban Art

\section{AUTHOR}

\section{ULRICH BLANCHÉ}

Ulrich Blanche est Docteur en Histoire de l'art et chercheur en Street Art depuis 2006. En 2010, il publie son premier livre « Street Artivist Banksy », puis en 2012 « Konsum Kunst ». Kultur und Kommerz bei Banksy und Hirst ». La traduction anglaise de ce dernier paraîtra en Janvier 2016 sous le titre « Banksy - Urban Art in a Material World». Blanché est professeur assistant au Département d'histoire de l'art de l'Université de Heidelberg en Allemagne.

Dr. Ulrich Blanché is a street art researcher since 2006. In 2010 he published his first book "Street Artivist Banksy », in 2012 « Konsumkunst. Kultur\&Kommerz bei Banksy und Hirst », which is going to be published in English in January 2016 as « Banksy - Urban Art in a material World ». Blanché is an assistant professor at Heidelberg University, Germany, department of art history. 